\title{
Statistical modelling for prediction of axis-switching in rectangular jets
}

\author{
T J Tipnis ${ }^{1}$, K Knowles ${ }^{2}$ and D Bray ${ }^{2}$
}

\begin{abstract}
Rectangular nozzles are increasingly used for modern military aircraft propulsion installations, including the roll nozzles on the F-35B V/STOL strike fighter. A peculiar phenomenon known as axis-switching is generally observed in such non-axisymmetric nozzle flows during which the jet spreads faster along the minor axis compared to the major axis. This might affect the under-wing stores and aircraft structure. A computational fluid dynamics study was performed to understand the effects of changing the upstream nozzle geometry on a rectangular free jet. A method is proposed, involving the formulation of an equation based upon a statistical model for a rectangular nozzle with an exit aspect ratio $\left(A R_{e}\right)$ of 4 ; the variables under consideration (for a constant nozzle pressure ratio $(N P R)$ ) being inlet aspect ratio $\left(A R_{i}\right)$ and length of the contraction section. The jet development was characterised using two parameters: location of the cross-over point $\left(X_{c}\right)$ and the difference in the jet half-velocity-widths along the major and minor axes $\left(\Delta B_{30}\right)$. Based on the observed results, two statistical models were formulated for the prediction of axis-switching; the first model gives the location of the cross-over point, while the second model indicates the occurrence of axis-switching for the given configuration.
\end{abstract}

\section{Keywords}

Axis-switching, CFD, secondary flows, rectangular nozzles

\section{Nomenclature}

$A_{e} \quad$ Area of the nozzle exit $\left(m^{2}\right) ; A_{e}=b \times h$

$A R_{e} \quad$ Aspect ratio of the nozzle exit; $A R_{e}=b / h$

$A R_{i} \quad$ Aspect ratio of the nozzle inlet; for rectangular inlets, it is the ratio of the width to the height of the inlet

$b \quad$ Width of the rectangular nozzle exit $(m)$

${ }^{1}$ Department of Engineering Photonics, Cranfield University, Cranfield, UK.

${ }^{2}$ Aeromechanical Systems Group, Cranfield University, Defence Academy of the UK, Shrivenham, UK.

Corresponding Author:

Kevin Knowles, Head, Aeromechanical Systems Group, Cranfield University, Defence Academy of the UK, Shrivenham, SN6 8LA, UK. Email: k.knowles@cranfield.ac.uk
$B \quad$ Jet half-velocity-width, in the direction indicated by the subscript $(m)$

$D_{e q} \quad$ Nozzle equivalent diameter $(m) ; D_{e q}=$ $\sqrt{4 b h / \pi}$

$h \quad$ Height of the rectangular nozzle exit $(m)$

$M_{j} \quad$ Jet Mach number

Re Reynolds number; $R e=\rho V d / \mu$

$V_{x} \quad$ Streamwise velocity $(\mathrm{m} / \mathrm{s})$

$V_{\text {exit }} \quad$ Centreline streamwise velocity at nozzle exit $(\mathrm{m} / \mathrm{s})$

$x, y, z \quad$ Cartesian coordinates, $x$ indicates streamwise direction, $y$ and $z$ are along minor and major axes, respectively 
$X_{c} \quad$ Cross-over point, streamwise position downstream of nozzle exit where jet halfvelocity-widths are equal $(m)$

$\Delta B_{30} \quad$ Difference in half-velocity widths along major and minor axes $(m) ; \Delta B_{30}=B_{z}-B_{y}$

$\omega_{x} \quad$ Streamwise vorticity $(/ s)$

\section{3-D Three-dimensional}

CFD Computational fluid dynamics

CSR Converging section ratio; ratio of length of converging section to length of nozzle

LDA Laser Doppler anemometry

NPR Nozzle pressure ratio; ratio of nozzle total pressure to ambient static pressure

V/STOL Vertical/Short take-off and landing

\section{Introduction}

The development of a free jet issuing from a circular nozzle has been studied extensively over the past many years, mainly for aircraft propulsion purposes. The flow structure and behaviour is generally well understood. More recently, investigations into the development of nonaxisymmetric free jets have also been undertaken though this area remains relatively less well understood than its circular counterpart. Current advances in modern warfare often require fighter aircraft to be designed to be stealthy and highly manoeuvrable. A rectangular jet generally provides better jet mixing, thus reducing the jet infra-red (IR) signature and thus improving the stealth of the aircraft. At the same time, it is easier to incorporate thrust vectoring along a single dimension when using a rectangular nozzle. This improves the manoeuvrability of the aircraft. Rectangular nozzles are increasingly used in military aircraft propulsion installations, including the roll nozzles on the $F-35 B \mathrm{~V} / \mathrm{STOL}$ strike fighter.

In general, it is known that the flow development of a jet depends upon various factors such as the nozzle pressure ratio $(N P R)$, exit area $\left(A_{e}\right)$, length of the nozzle contraction, aspect ratio $(A R)$, etc. It has been shown by various authors $[1,2,3]$ that the incompressible rectangular free jet consists of three regions: the potential core region with constant centreline velocity, a two-dimensional region and a region extending to infinity where the centreline velocity decay is characteristic of axisymmetric jets. The extent of these regions is a function of the nozzle exit aspect ratio. Some of these studies also report a peculiar phenomenon usually associated with rectangular and non-axisymmetric nozzles, often called axis-switching. This does not occur due to a helical turning of the jet but is, in fact, caused by different spreading rates along the major and minor axes of the jet. Thus, it appears as if the jet has turned at right angles some distance downstream of the exit $[4,5]$.

Many studies have attempted to explain this. Early studies suggested that the occurrence of axis-switching is dominated by the dynamics of vortex rings and selfinduced axis-rotations of the vortex rings. Abramovich [6] suggested that axis-switching is due to pressure differentials on the plane of the rectangular vortex ring, caused by the difference in the separations of the long and the short edges of the ring. This leads to self-induced induction, as also observed by Hussain and Husain [7]. The most important amongst these explanations for axisswitching are based on vorticity and the presence of discrete vortical structures in the jet $[8,5,9]$. Work carried out independently by Zaman [10] and Grinstein [11] 
for non-axisymmetric nozzles with circular inlets indicates that axis-switching might not occur at all. It was thus suggested that the vortices either form an 'out-flow' pair or an 'in-flow' pair at the ends of the major axis. The pair of vortices which appear to push the fluid away from the centre, thus resisting axis-switching, was called the 'out-flow' pair. Similarly, the pair of vortices that appears to push the fluid towards the centre (and thus away from the centre along the minor axis) seems to assist axisswitching. This was referred to as an 'in-flow' pair. The occurrence of axis-switching thus depends on the presence of vortical structures in the flow. The secondary flows inside the nozzle could result in the presence of streamwise vorticity at the jet exit and could play an important role in the development of the jet [10].As the flow develops in the interior of the nozzle, the pressure differential along the walls of the nozzle induces secondary flows in the plane perpendicular to the streamwise flow [12]. The variation of the cross-over point with $A R_{e}>$ 5 has also been shown previously $[1,13,14,15]$. These studies show a linear variation but the different studies reveal a range of slopes rather than a specific one, indicating uncertainty in determining the location of the cross-over point. Additionally, Krothapalli, et al. [15] discuss the linear variation of jet-half-velocity on initial geometries but it is unclear as to exactly which is the dominant parameter. They also observed that a jet issuing from a slot exhibits axis-switching sooner compared to one from a long channel feeding a similar contraction ratio.

It can thus be seen that amongst all the factors that affect the development of the jet, the variation due to specific changes in the internal geometry of the nozzle has not been documented systematically. It has been estab- lished that the internal flow affects the development of the flow outside the nozzle. There remains, however, a need to understand the relation between internal geometry factors such as the inlet aspect ratio $\left(A R_{i}\right)$ and the length of the contraction section with respect to axis-switching. A good understanding of this can lead to the development of methods for the prediction of axis-switching for a given set of conditions.

The enhanced entrainment properties of a non-circular jet relative to its circular counterpart are mainly attributed to the axis-switching mechanism. Besides its application for reducing the $I R$ signature of a heated jet plume, improved mixing of the jet with its surroundings has other potential uses. This property could be of interest in practical applications requiring enhanced combustion between fuel and background oxidiser, reduction in jet noise and rapid initial mixing and submergence of effluent fluids, to name a few [16].

This paper attempts to establish the variation of the cross-over point with the inlet aspect ratio and the length of converging section. The length of the converging section is expressed in a normalised form as converging section ratio (CSR), defined as the ratio of the length of the contraction section to the length of the nozzle.

\section{Computational setup}

The 3-D CFD simulations were performed using the commercial $C F D$ package Fluent; the grids were created using the Gambit pre-processing software. The simulations were carried out for a steady state, subsonic case with $N P R=1.05$, corresponding to $M_{j}=0.265$ and Reynolds number of about 59,700 (based on the nozzle height). This is within the range of the experimental studies by 


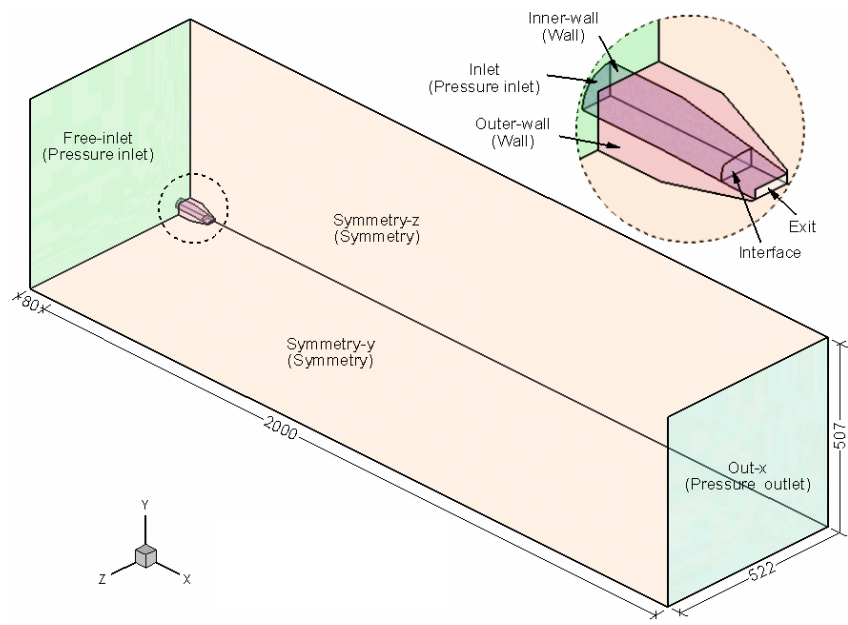

(a) Domain extents, orientation and boundary types for the simulations; the lateral pressure outlets (Out-y and Out-z) are not shown in the figure; dimensions are in $\mathrm{mm}$

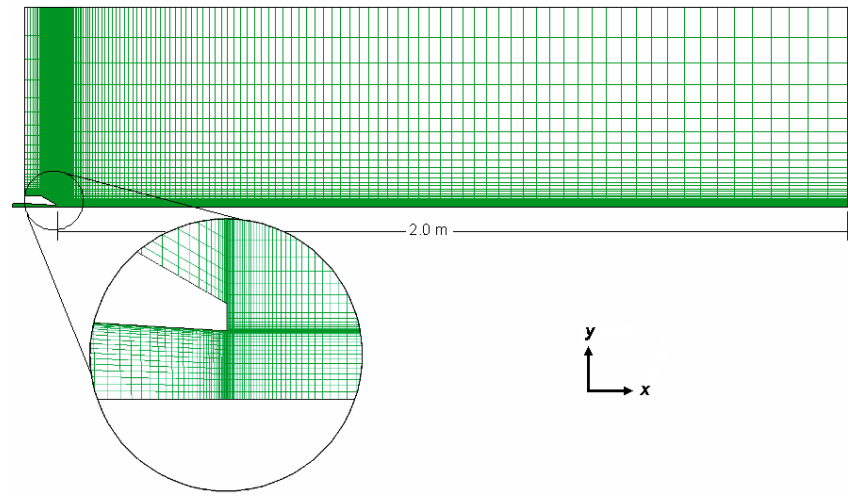

(b) Grid distribution and dimensions along the $x y$ symmetry plane

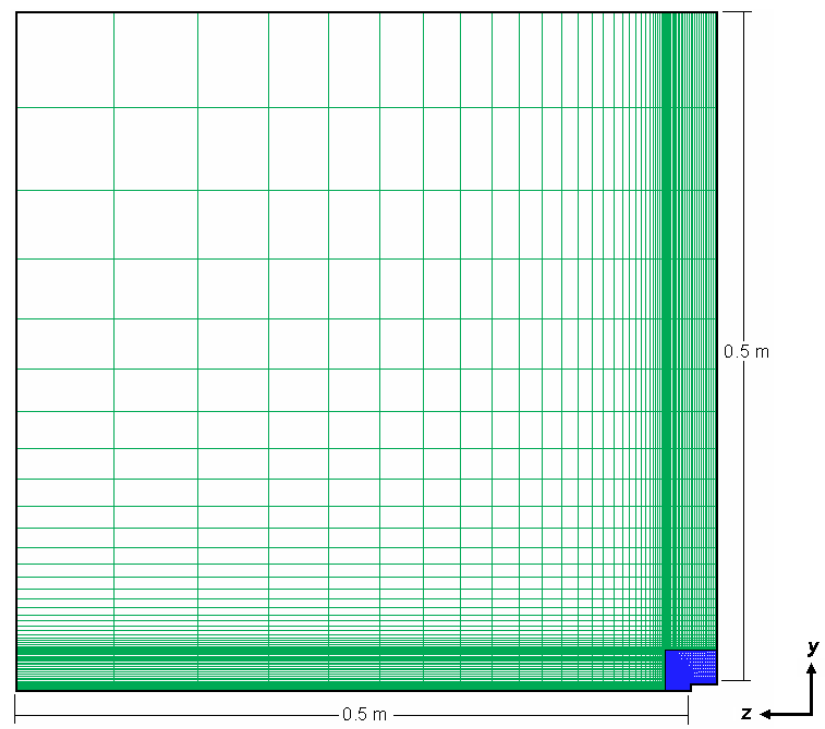

(c) Grid distribution and dimensions along the spanwise direction at the free inlet boundary

Figure 1: Computational domain and grid setup
Krothapalli [15] $(\operatorname{Re}=12,000$, laminar boundary layer $)$ and Sfeir [13] $(\operatorname{Re}=122,200$, turbulent boundary layer $)$ which are used for comparative purposes. Additionally, since the $C F D$ simulation assumed turbulent boundary layer on the nozzle walls, changing the scale of the nozzle was unlikely to produce any Reynolds number dependency on the $C F D$ results. Running the simulation in steady state allowed the use of symmetry conditions along the major and minor axis planes of the nozzle. The nozzle exit was $400 \mathrm{~mm}^{2}$ with $A R_{e}=4$. The nozzle length was 110 $\mathrm{mm}$ with an area contraction of approximately 2.4 ; representative of an existing nozzle with a circular inlet and contraction length of $65 \mathrm{~mm}$, which was used in experiments for validation purposes. The exterior dimensions of the nozzle configurations were also based upon the dimensions of this nozzle. The external domain of the simulation was $2 \mathrm{~m}$ downstream (approximately $90 D_{e q}$ ) of the exit and $0.5 \mathrm{~m}$ in the lateral direction from both the outer edges of the nozzle exit. In the upstream direction outside the nozzle, the domain extended as much as the exterior of the nozzle. A grid independence study was done to determine the optimum meshing for the domain. This was done using three different grids of approximately 460,000 cells (coarse), 650,000 cells (medium) and 820,000 cells (fine). The primary variation in the grid was the mesh distribution along the downstream and span-wise directions. Amongst these, the coarse grid indicated a shorter potential core length, while the medium grid was able to resolve the jet structures as well as the fine grid. The domain setup, orientation and the grid distribution along the $x y$-plane is shown in Figure 1. For all the simulations, care was taken to ensure that the boundary layer was resolved as correctly as possible. This was achieved by keeping the 
wall $y^{+}$values at the inner wall of the nozzle to between 1 and 10. The range of parameters used for the different nozzle configurations is given in Table 1 .

The simulation was set up as a compressible flow problem using a pressure based solver. SST-kw was chosen as the turbulence model since it can predict both wall flows and free flows with sufficient accuracy [17]. The turbulence was specified using intensity and length scale values. The turbulence intensity at the inlet was determined using the exit turbulence intensity from the experiments and then adjusted accordingly so that the simulation predicts the same exit turbulence intensity. The length scale was taken as $7.5 \%$ of the equivalent radius of the nozzle [18]. The inlet was specified as a pressure inlet at $N P R=$ 1.05. The upstream boundary outside the nozzle was specified as a pressure inlet at $N P R=1.00$ to allow free inflow as required by the simulation without imposing any limiting factor. The $x y$ - and $x z$-planes were specified as symmetry boundaries. The other outer boundaries were set as pressure outlets with free-stream pressure conditions.

Table 1: Range of variation for $A R_{i}$ and $C S R$; the name for the configuration is given in each cell

\begin{tabular}{||c|c|c|c|c||}
\hline \hline $\boldsymbol{C S R} \backslash A \boldsymbol{R}_{\boldsymbol{i}}$ & $\mathbf{1}$ & $\mathbf{2}$ & $\mathbf{3}$ & $\mathbf{4}$ \\
\hline $\mathbf{0 . 4 0}$ & $R A 1 C 1$ & $R A 2 C 1$ & $R A 3 C 1$ & $R A 4 C 1$ \\
\hline $\mathbf{0 . 5 9}$ & $R A 1 C 2$ & $R A 2 C 2$ & $R A 3 C 2$ & $R A 4 C 2$ \\
\hline $\mathbf{0 . 8 0}$ & $R A 1 C 3$ & $R A 2 C 3$ & $R A 3 C 3$ & $R A 4 C 3$ \\
\hline $\mathbf{1 . 0 0}$ & $R A 1 C 4$ & $R A 2 C 4$ & $R A 3 C 4$ & $R A 4 C 4$ \\
\hline \hline
\end{tabular}

To check the convergence of the simulation, four monitors were initiated. One of these monitors checked the mass flux for the entire domain (including the nozzle) and compared that with the net mass flow rate of the nozzle [19]. If the total mass flux was consistently less than $0.5 \%$ of the mass flow rate of the nozzle over a period of 3,000 iterations, convergence was assumed to have been achieved in terms of mass flux. The other three monit-
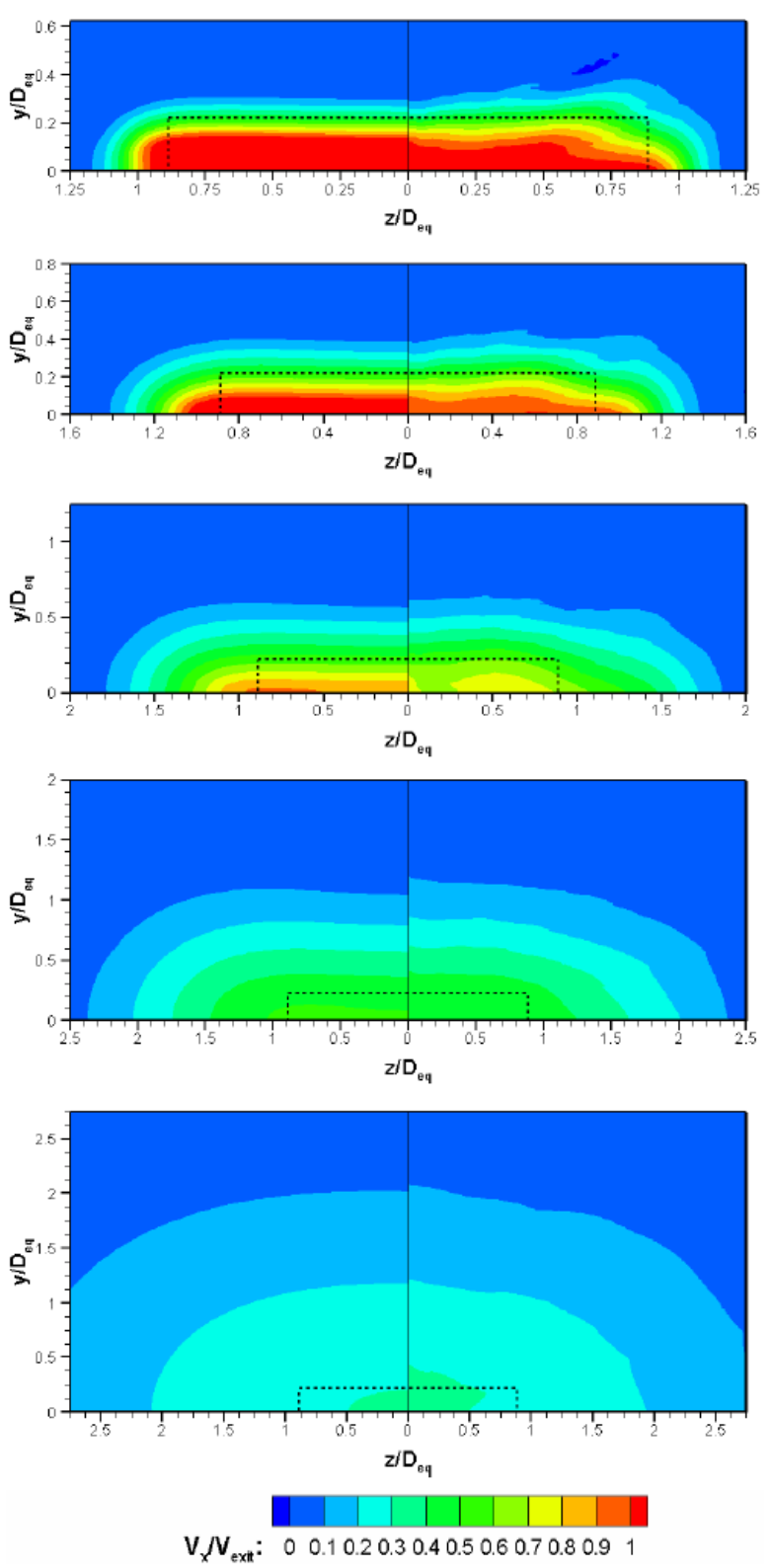

Figure 2: Comparison of normalised streamwise velocity contours $\left(V_{x} / V_{\text {exit }}\right)$ at planes downstream of the exit; from top to bottom, at 1 , 2, 4, 8 and $16 D_{e q}$; CFD simulation results (left); $L D A$ experiment results (right); results shown only for one quadrant of the nozzle each, the dotted outline indicates the dimensions of the nozzle exit

ors checked the velocity at three points downstream of the nozzle exit at $x / D_{e q}=2,25$ and 50, each. If the variation of velocity at these points was less than $0.5 \%$, averaged over 3000 iterations, velocity convergence was assumed to have been achieved.

The validation of the $C F D$ results was done using data from an $L D A$ experiment for the same nozzle at the given flow parameters and conditions [19]. The axial velocities 
at five different downstream locations was compared, as shown in Figure 2. It can be seen that the velocity development downstream of the exit shows generally good agreement, particularly in terms of the jet vertical and lateral spreading. Following this, the simulations were modelled to study the other inlet aspect ratios.

\section{Results and discussion}

The two main parameters considered for this study are the normalised location of the cross-over point $\left(X_{c} / D_{e q}\right)$ and the normalised difference between the spreading of the jet along the major and minor axes at $x / D_{e q}=30$ $\left(\Delta B_{30} / D_{e q}\right)$. The cross-over point is defined as the location downstream of the nozzle exit where the half-velocitywidths along the lateral axes are equal. Where the jet does not exhibit axis-switching, the transition point, i.e. the location downstream of the exit where the spreading rates along the lateral axes are equal and the jet shows a transition to an elliptic/round jet cross-section, is used in the formulation of the statistical model.

The simulation results were compared for all the nozzle configurations under consideration. The location of the cross-over point and difference between the spreading of the jet along the major and minor axes at $x / D_{e q}=30$, were noted. These were then used for the formulation of the statistical models for prediction of axis-switching. The possible explanations for the occurrence of axisswitching, or otherwise, have been discussed based on streamwise vorticity development due to secondary flows in the nozzle. The plots given here are for the $C 2$ (CSR $=0.59)$ and $A 3\left(A R_{i}=3\right)$ configurations to illustrate the trends based on changing one parameter, either $A R_{i}$ or CSR, keeping the other constant.

\subsection{Jet half-velocity-widths}

The basis for determining if the jet issuing from a nozzle of given configuration switches axes, or not, was to compare the jet half-velocity-width $(B)$. This is defined as twice the distance from the centreline of the jet to where the local streamwise velocity is equal to half of the local centreline streamwise velocity. Henceforth in this work, jet half-velocity-width will be used in the normalised form (i.e. $B / D_{e q}$ ). Since the concept of this work was inspired by the potential issues of the $F-35 B$ V/STOL strike fighter, the extent of the region of interest was considered to be up to $x / D_{e q}=30$. Consequently, the half-velocity-width plots for the current study are generated up to $x / D_{e q}=30$. Additionally, the difference between the jet-widths along the major and minor axes, at a distance of $x / D_{e q}=30$ was also recorded and used as the other parameter to identify axis-switching $\left(\Delta B_{30} / D_{e q}\right)$.

The streamwise variation of the jet half-velocity-widths for the $C 2$ and $A 3$ configurations are given in Figures 3 and 4 , respectively. It can be seen that the jets issuing from a nozzle of $A R_{i}=1$ do not switch axes. These jets assume equal spreading rates at a certain distance downstream of the exit. The data obtained from these plots, i.e. the locations of the cross-over point $\left(X_{c}\right)$ and the difference in the jet half-velocity-widths $\left(\Delta B_{30} / D_{e q}\right)$, for all the 16 configurations, are presented in Table 2. In the case of the $A R_{i}=1$ configurations, $X_{c}$ indicates the location where the jet achieves approximately equal spreading rates along the major and minor axes. The negative values in Table 2(b) indicate occurrence of axis-switching before $x / D_{e q}=30$, while positive values indicate that axis-switching does not occur.

The data from Table 2(a) were compared to the exper- 

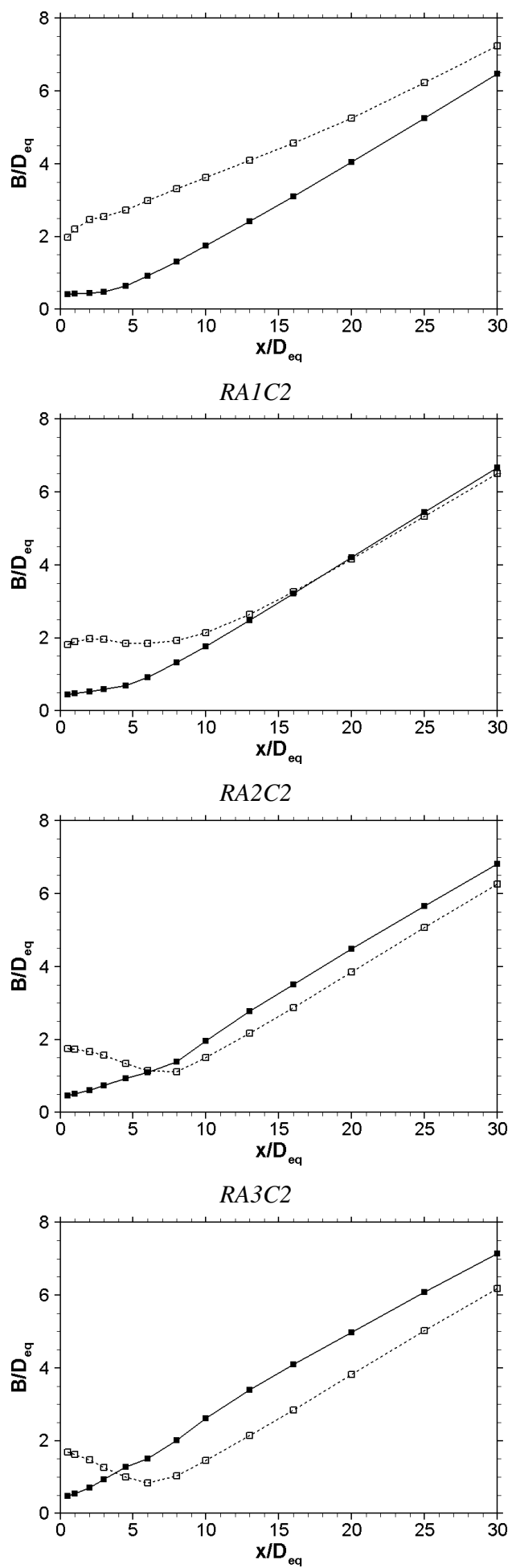

$R A 4 C 2$

Figure 3: Streamwise variation of jet half-velocity-width, constant $C S R:-\square-, B_{y} / D_{e q} ;--\square--, B_{z} / D_{e q}$

imental results from Krothapalli, et al.(1981) [15]. If the region indicating the variation of cross-over point is extrapolated back towards the lower aspect ratios, the values
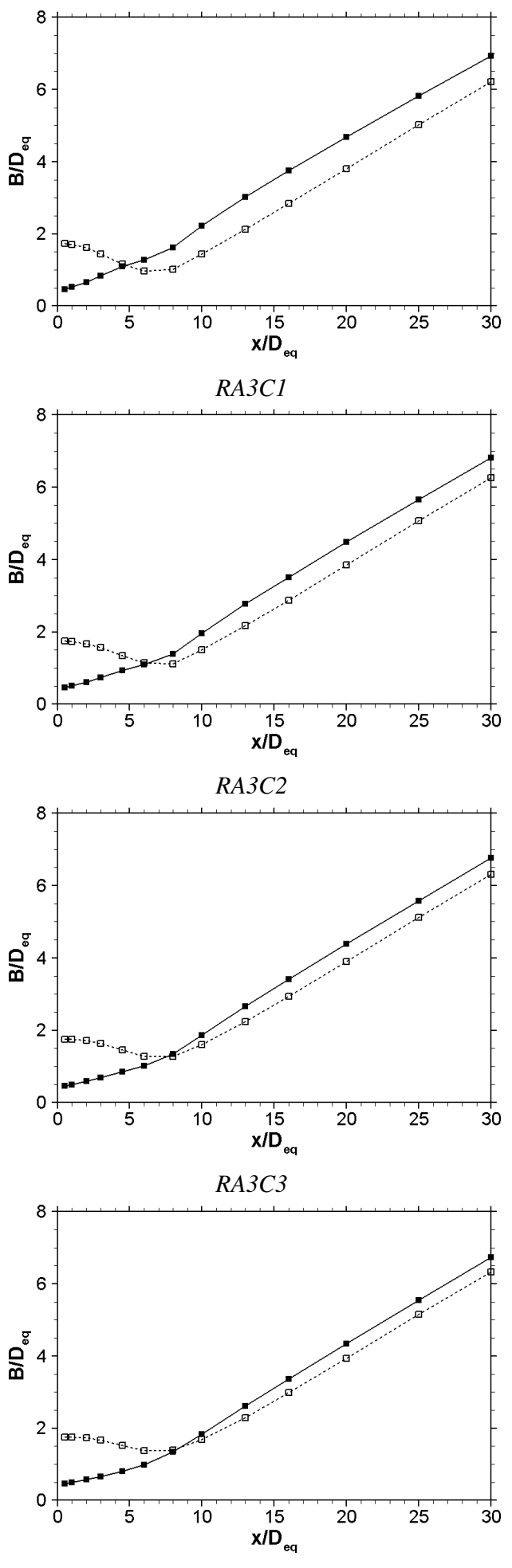

$R A 3 C 4$

Figure 4: Streamwise variation of jet half-velocity-width, constant $A R_{i}:-\square-, B_{y} / D_{e q} ;--\square--, B_{z} / D_{e q}$

obtained from the $C F D$ simulations lie within the extended region, as seen in Figure 5. It can, therefore, be said that the locations of the cross-over point given by the sim- 
Table 2: Data obtained from the half-velocity-width plots generated for the different configurations

\begin{tabular}{||c|c|c|c|c||}
\hline \hline${\boldsymbol{C S R} \backslash \boldsymbol{A R} \boldsymbol{R}_{\boldsymbol{i}}}$ & $\mathbf{1}$ & $\mathbf{2}$ & $\mathbf{3}$ & $\mathbf{4}$ \\
\hline $\mathbf{0 . 4 0}$ & 51.8169 & 17.5091 & 4.7780 & 2.7942 \\
\hline $\mathbf{0 . 5 9}$ & 48.0521 & 17.7100 & 6.2531 & 3.7933 \\
\hline $\mathbf{0 . 8 0}$ & 44.1317 & 17.9032 & 7.5502 & 4.7911 \\
\hline $\mathbf{1 . 0 0}$ & 39.6184 & 18.0566 & 8.4929 & 5.6953 \\
\hline \hline
\end{tabular}

(a) Location of the cross-over point $\left(X_{c} / D_{e q}\right)$

\begin{tabular}{||c|c|c|c|c||}
\hline \hline $\boldsymbol{C S R} \backslash A \boldsymbol{R}_{\boldsymbol{i}}$ & $\mathbf{1}$ & $\mathbf{2}$ & $\mathbf{3}$ & $\mathbf{4}$ \\
\hline $\mathbf{0 . 4 0}$ & 1.3127 & -0.1642 & -0.7251 & -1.5479 \\
\hline $\mathbf{0 . 5 9}$ & 0.7759 & -0.1657 & -0.5526 & -0.9684 \\
\hline $\mathbf{0 . 8 0}$ & 0.4496 & -0.1674 & -0.4613 & -0.7165 \\
\hline $\mathbf{1 . 0 0}$ & 0.3080 & -0.1692 & -0.3949 & -0.5893 \\
\hline \hline
\end{tabular}

(b) Difference between the jet-widths along the major and minor axes $\left(\triangle B_{30} / D_{e q}\right)$ at $x / D_{e q}=30$

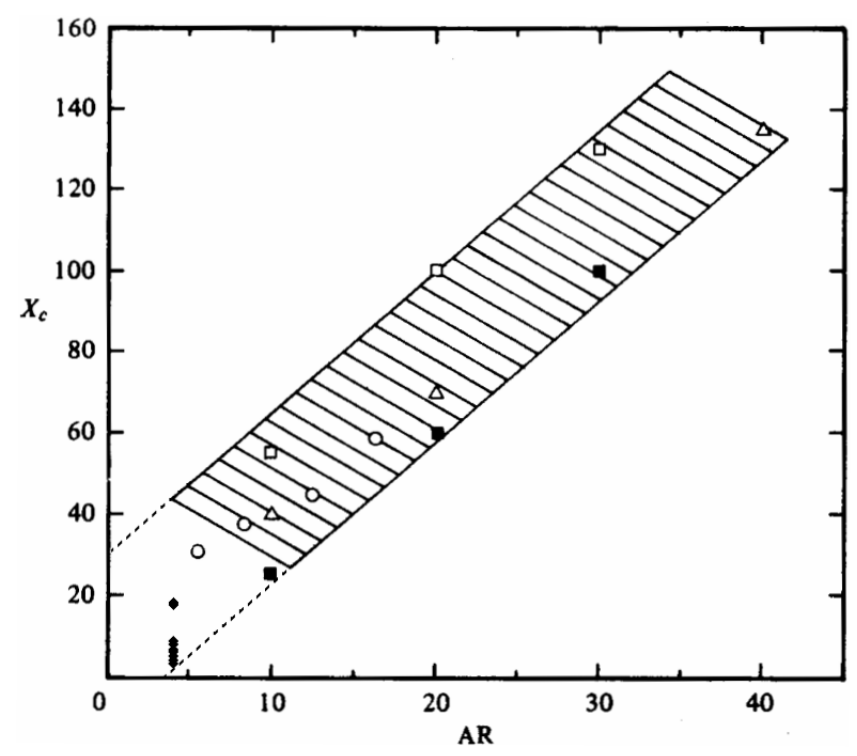

Figure 5: Validation of cross-over point locations obtained using CFD data, the range is extrapolated back towards lower aspect ratios; original data from Krothapalli, et al. [15]; O, Krothapalli, et al. [15]; $\triangle$, Sforza, et al. [1]; $\square, \mathbf{\square}$, Sfeir [13] (filled square indicates orifice jet); $\diamond$, current $C F D$ results

ulations were within the expected range.

\subsection{Streamwise vorticity development}

The different nozzle configurations, as viewed from the downstream direction, are shown in Figure 6. The stream-

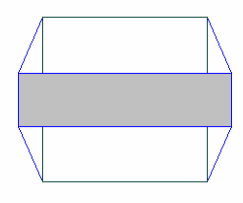

$R A 1$

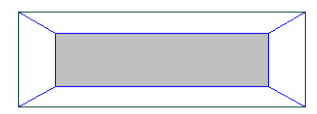

$R A 3$

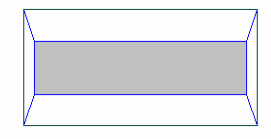

$R A 2$

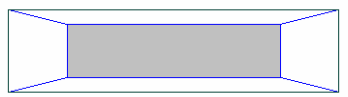

$R A 4$
Figure 6: Front view of the configurations based on different inlet aspect ratios; the shaded area indicates nozzle exit

wise vortices, developing from these nozzle are observed at two downstream locations, $x / D_{e q}=2$ and 8 . Figure 7 shows the normalised streamwise vorticity $\left(\omega_{x} D_{e q} / V_{e x i t}\right)$ and the normalised streamwise velocity $\left(V_{x} / V_{\text {exit }}\right)$ contour plots.

The change in nozzle geometry affects the development of the free jet, especially the unequal spreading rates that may or may not result in axis-switching. The unequal spreading rates may be due to the presence of secondary flows through the nozzle, which arise due to the change in the geometry. The change in the cross-section induces a difference in the acceleration of the flow along the shorter and the longer sides. The flow along the sides, which have a higher slope during the transition from the inlet to the exit geometry, tends to accelerate faster. Along any crosssection inside the nozzle, the viscous forces exerted normal to the wall surface due to the fluid are equal at any point for the given cross-section. Thus, the higher inertial forces generated along the side with a higher slope imply that the boundary layer thickness is smaller. The flow, therefore, leads to the production of secondary flows in the plane such that the fluid is pushed inwards from 
the side which exhibits faster flow towards the centreline of the nozzle and then outwards to the side exhibiting slower flow and back along the walls of the nozzle. Such a secondary motion is thus conducive to the formation of streamwise vorticities. The presence of a diverging wall, therefore induces the formation of an 'out-flow' pair [20] while converging wall sections generally induce the formation of an 'in-flow' pair [21].

Indeed, for the $A R_{i}=1$ configurations, the flow accelerates faster along the ends of the minor axis compared to the major axis. This induces a secondary flow that pushes the fluid towards the centre of the nozzle from the minor axis ends and outwards towards the major axis end, i.e. in a clockwise sense. Such a flow tends to produce streamwise vorticity pairs ('out-flow' pairs) such that they push the fluid outwards from the ends of the major axis and inwards from the ends of the minor axis. This would resist the axis-switching process. The difference in the flow accelerations along the major and minor axes is very small for the $A R_{i}=2$ configurations; this can explain the delayed axis-switching encountered for these configurations. The $A R_{i}=3$ and 4 configurations, however, show a distinctly higher sloping of the nozzle walls along the major axis compared to the minor axis. Consequently, the flow acceleration is higher along the walls at the ends of the major axis and it induces a secondary flow in the counterclockwise sense, i.e. from the ends of the major axis towards the centre, on to the ends of the minor axis and back along the nozzle wall. This type of flow produces the 'inflow' pairs which tend to assist axis-switching.

The change in the CSR of the nozzle affects the slope of the nozzle walls along the transition section from the inlet to the exit geometry. Although this does not influence the sense of the streamwise vorticity, it affects the strength of the vorticities developed. Clearly, for lower CSR values, the slope of the nozzle walls is higher than that for higher $C S R$ values. The flow developing along the walls, therefore, tends to accelerate faster and thus induces secondary flows of higher magnitude. This is confirmed by the location of the cross-over point; the configurations with lower $C S R$ switch axes earlier. In case of the $A l$ set of nozzle configurations as well, where a lower CSR corresponds to a delay in transition or axis-switching, the observations can be explained due to the strength of the vortices generated; since the vortex pairs for these configurations tend to resist axis-switching, the stronger vortices generated for lower CSR configurations delay the transition or axisswitching. As the strength of the vortex field decreases with an increase in the $C S R$ value, the location at which the transition to an elliptic/round jet cross-section with equal spreading rates occurs moves closer to the nozzle exit with increasing $C S R$.

\subsection{Statistical model}

Based on the results presented in Table 2, two statistical models were created: 'XOP-model' for determining the location of the cross-over point, and 'DB30-model' for determining the occurrence of axis-switching. The creation of the model was a two-step process: a singlevariable model was created for each and depending upon the best-fit series of variation, a two-variable model was created to predict the necessary parameters. A rigorous error analysis implementing normality testing was done to ensure that the values predicted by the models were as close to those obtained by the simulation results used to create these models. The equations defining the two statistical models thus created, are given below: 

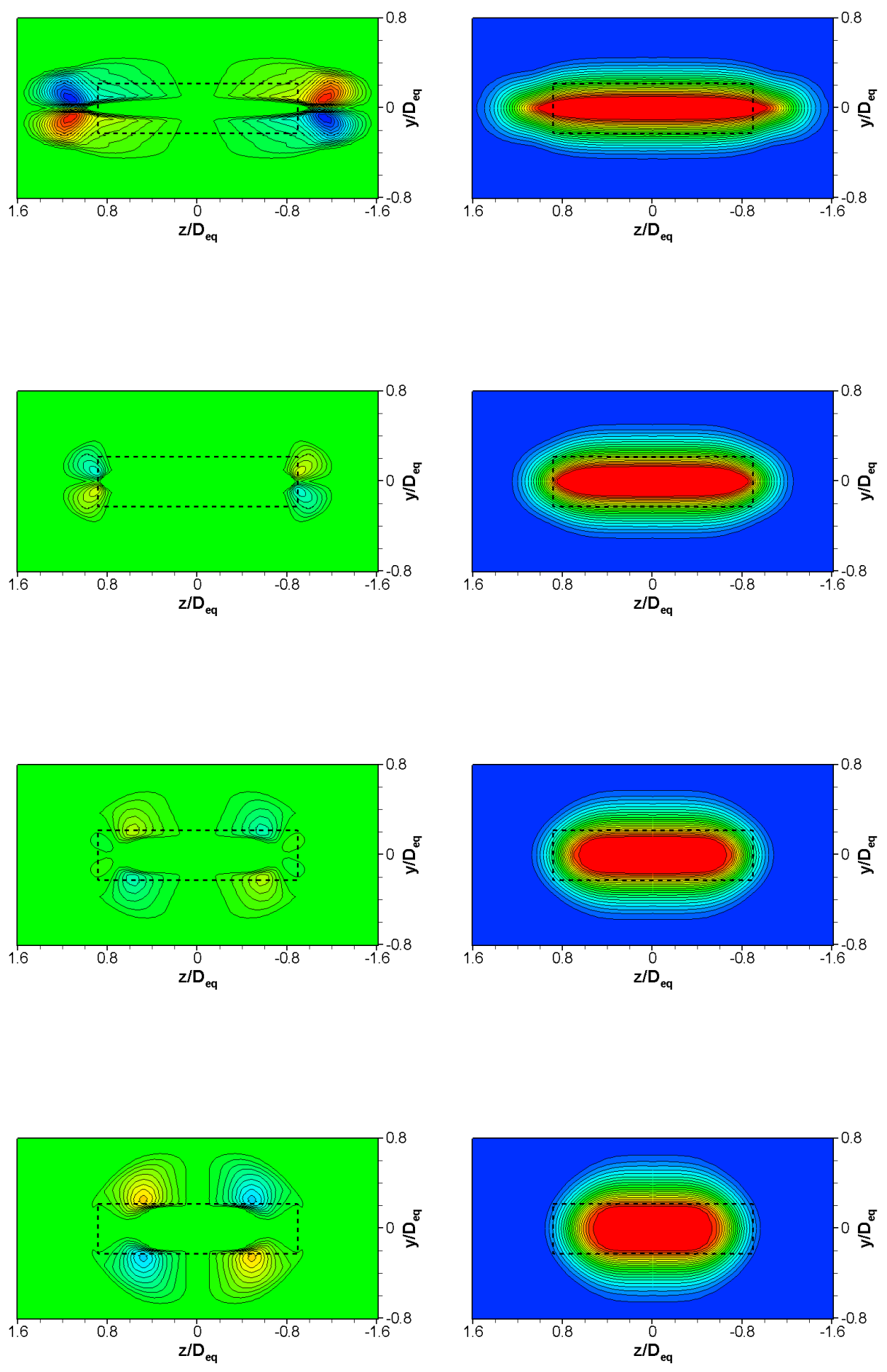

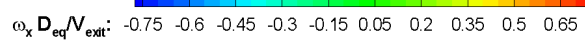

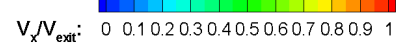

(a) CSR constant, $x / D_{e q}=2$; from top to bottom, $A R_{i}=1,2,3,4$

Figure 7: Contours of normalised streamwise vorticity $\left(\omega_{x} D_{e q} / V_{\text {exit }}\right)$ (left) and normalised streamwise velocity $\left(V_{x} / V_{\text {exit }}\right)($ right $)$ at planes $x / D_{e q}=$ 2 and 8; the dashed outline represents the nozzle exit 

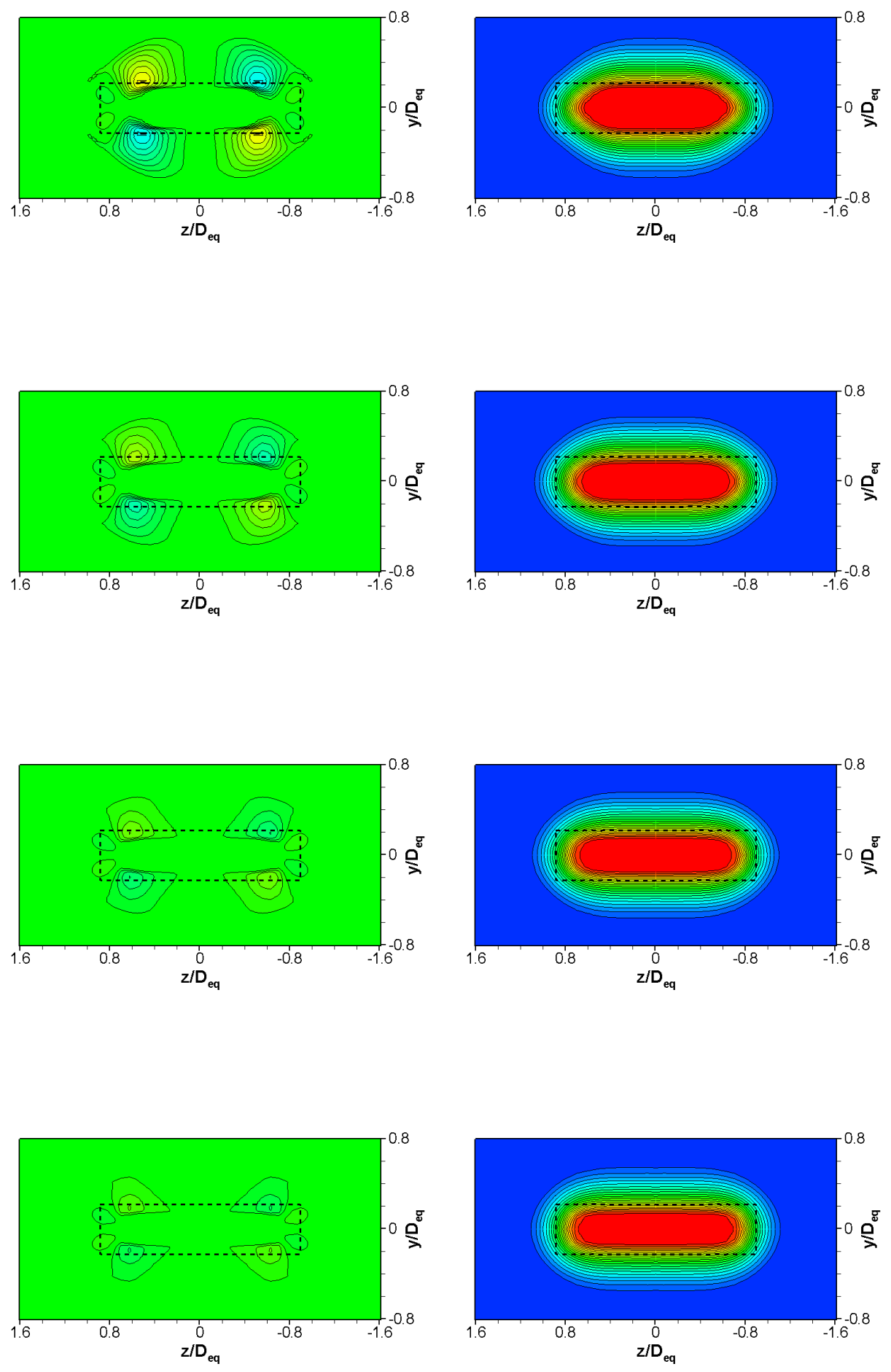

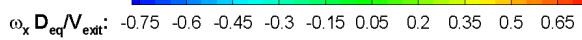

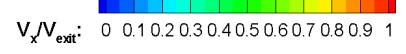

(b) $A R_{i}$ constant, $x / D_{e q}=2$; from top to bottom, $C S R=0.40,0.59,0.80,1.00$ 

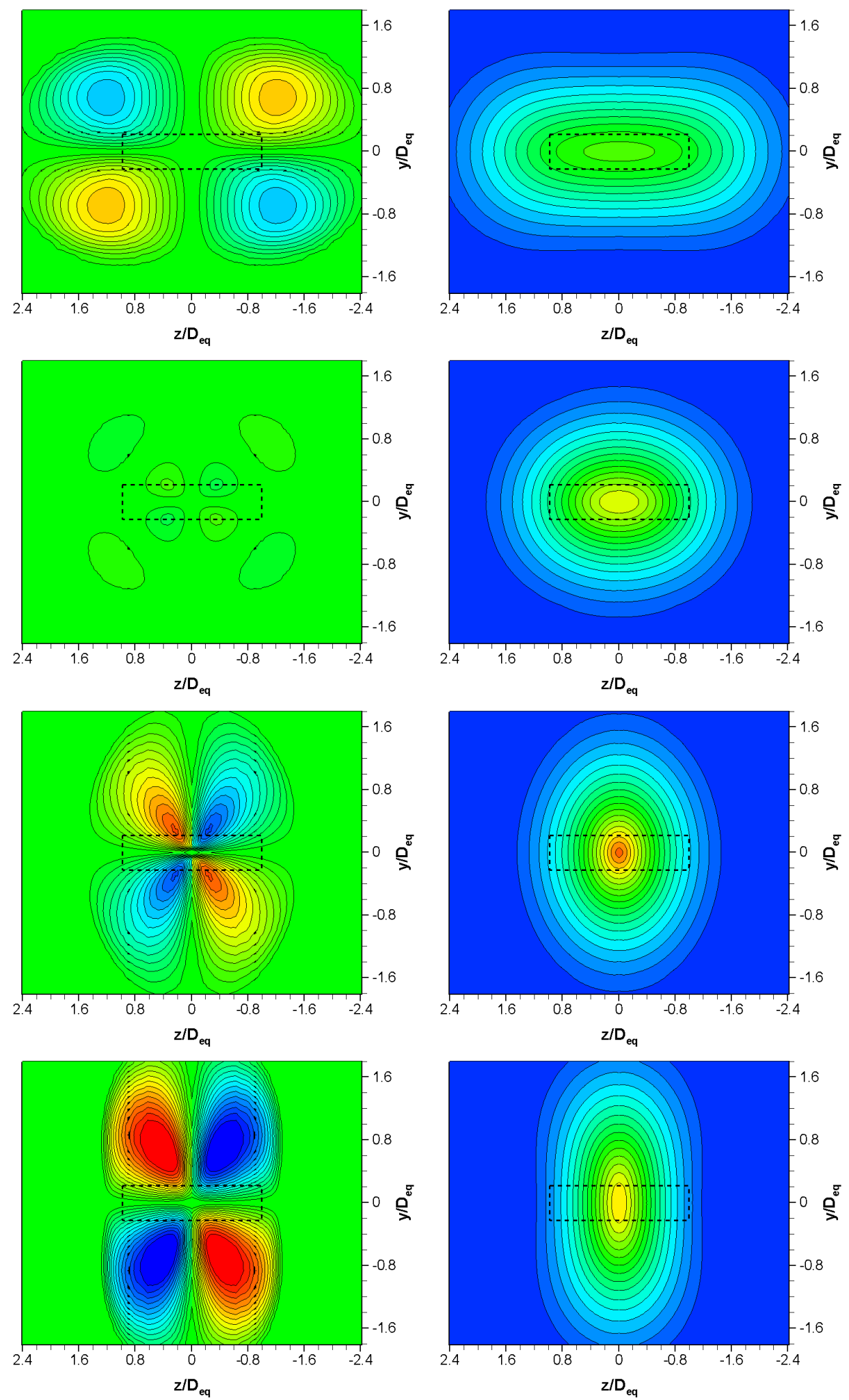

$\omega_{\mathrm{x}} \mathrm{D}_{\text {ed }} N_{\text {exit }}:-0.075-0.06-0.045-0.03-0.0150 .0050 .02 \quad 0.0350 .050 .065$

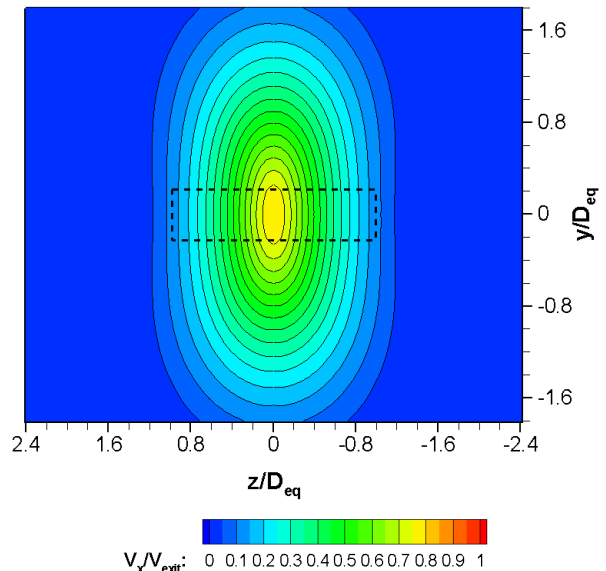

(c) $C S R$ constant, $x / D_{e q}=8$; from top to bottom, $A R_{i}=1,2,3,4$

Figure 7: Continued 

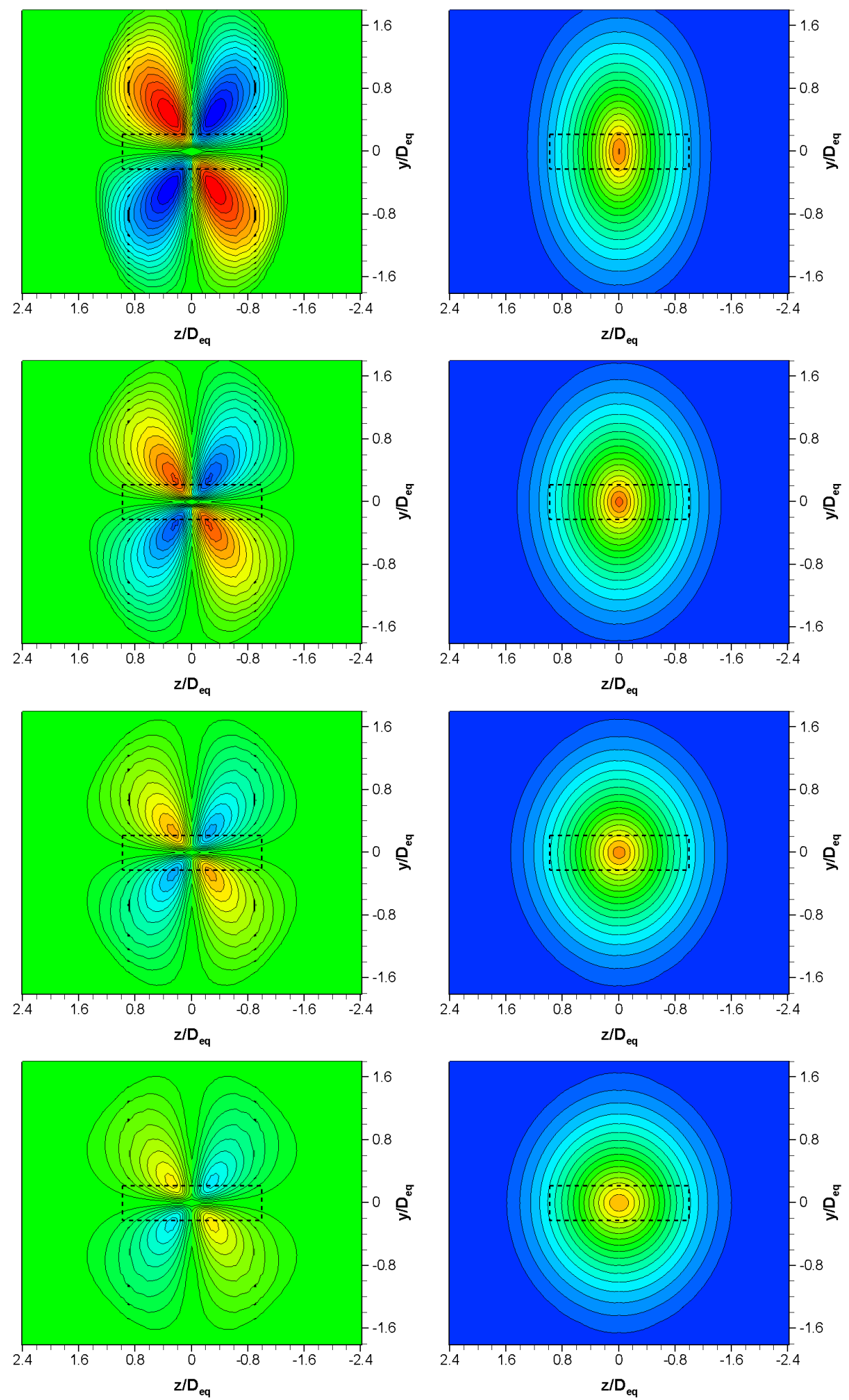

$\omega_{\mathrm{x}} \mathrm{D}_{\text {eq }} N_{\text {exit }}:-0.075-0.06-0.045-0.03-0.0150 .0050 .020 .0350 .050 .065$

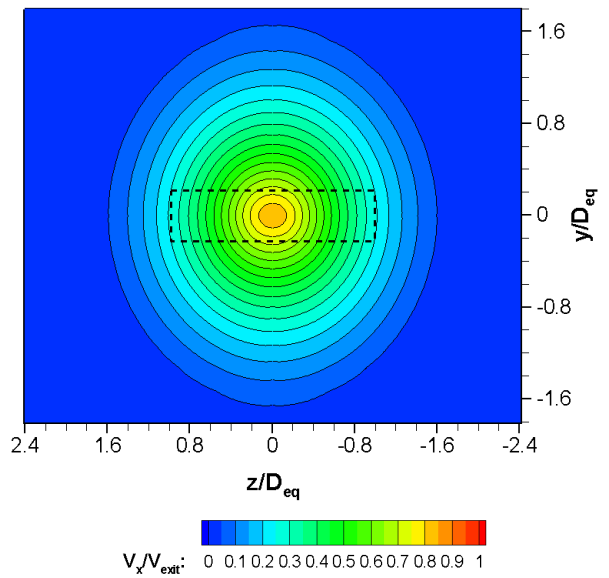

(d) $A R_{i}$ constant, $x / D_{e q}=8$; from top to bottom, $C S R=0.40,0.59,0.80,1.00$

Figure 7: Continued 


$$
\begin{gathered}
X_{c}=\left(\begin{array}{c}
1.5285 A R^{3}-17.0702 A R^{2} \\
+61.5803 A R-66.1947
\end{array}\right) C S R+ \\
\left(\begin{array}{c}
-2.4923 A R^{3}+29.0058 A R^{2} \\
-112.3766 A R+145.8268
\end{array}\right) \\
\Delta B_{30}=-\left(0.0345 C S R^{-1.8947}\right) A R_{i}^{3} \\
+\left(0.3291 C S R^{-1.7507}\right) A R_{i}^{2} \\
-\left(1.2192 C S R^{-1.5463}\right) A R_{i} \\
+\left(1.2414 C S R^{-1.5084}\right)
\end{gathered}
$$

Both the equations use dimensionless values for input $\left(A R_{i}\right.$ and $\left.C S R\right)$ and give the required output in a normalised form for $X_{c}$ and $\Delta B_{30}$. The XOP and DB30 models could now be used for the $A R_{e}=4$ nozzles in the current work to determine if they switch axes and, if so, the location of the cross-over point downstream of the exit (Figure 8). In cases where the model indicates that a particular configuration does not switch axes, the location of the cross-over point corresponds to the point where the jet spreading along the spanwise axes becomes almost equal.

\section{Conclusions}

$C F D$ simulations for a series of nozzle configurations based on $A R_{e}=4$ were carried out to understand the effect of changing the upstream nozzle geometry on the development of a rectangular free jet. The parameters under consideration were the inlet aspect ratio and length of the contraction section.

The nozzle configurations with $A R_{i}=1$ do not exhibit axis-switching. For a constant inlet aspect ratio, the variation of the location of the cross-over point $\left(X_{c}\right)$ is roughly linear with respect to the converging section ratio. A higher $C S R$ corresponds to a higher $X_{c}$ for the axisswitching cases while it is inversely proportional for the

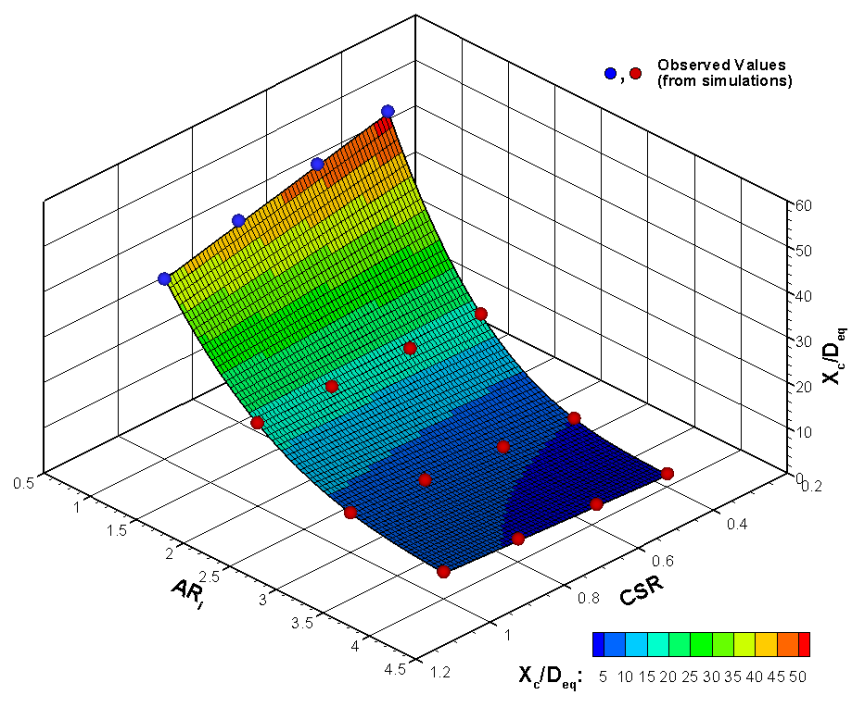

(a) 'XOP-model' for location of cross-over point; the observed values from the $C F D$ simulations are indicated by red circles for cross-over points and by blue circles for transition points

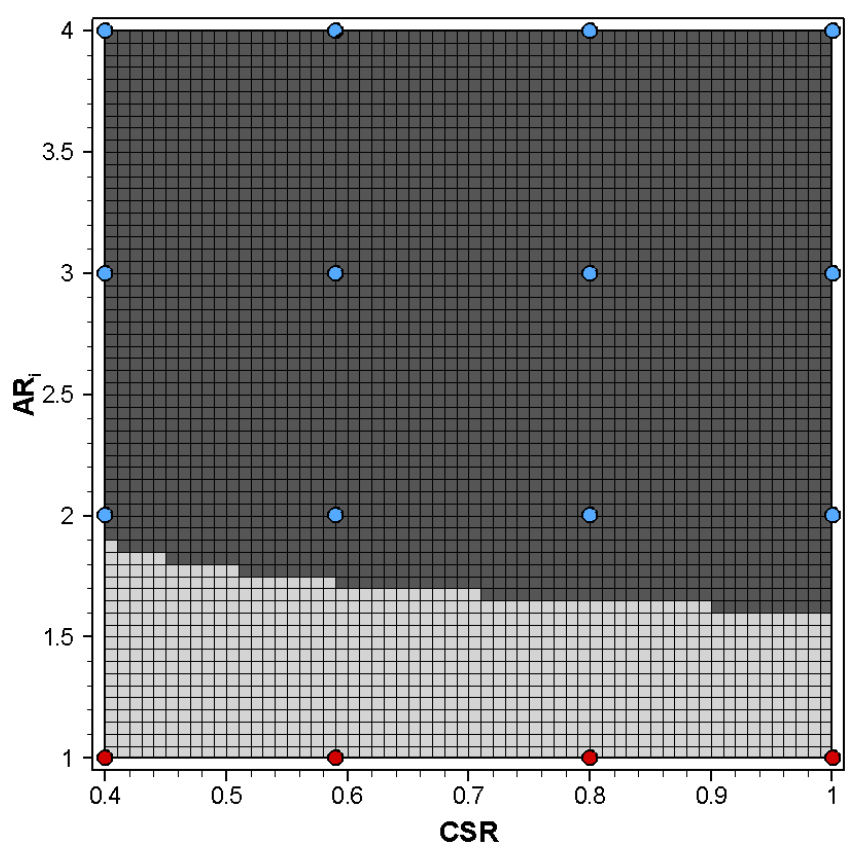

(b) 'DB30-model' for determining the occurrence of axis-switching; dark squares indicate occurrence of axis-switching before $x / D_{e q}=30$, light squares indicate otherwise; $C F D$ results are shown in blue circles indicating axis-switching cases, and in red circles to indicate otherwise

Figure 8: Graphical representation of the predicted values based on the statistical models created for the rectangular nozzle configurations

non-axis-switching configurations. The converging section ratio remaining constant, the $X_{c}$ varies inversely with respect to $A R_{i}$, i.e. lower $A R_{i}$ values correspond to higher $X_{c}$ values. The variation of $\Delta B_{30}$, on the other hand, is 
non-linear with respect to both $A R_{i}$ and $C S R$.

The difference in the flow acceleration along the side walls of the nozzle induces secondary flows which are responsible for the generation of streamwise vorticity. Depending on the development of these vortices, these may assist or resist axis-switching, indicated by the presence of an 'out-flow' or 'in-flow' pair at the ends of the nozzle major axis. While the sense of the streamwise vorticity is mainly dependent on the $A R_{i}$ of the nozzle, the magnitude of the vorticity in the vortex core is mainly dependent on the $C S R$ of the nozzle.

While the current study focusses primarily on development of a parametric model based on the axis-switching phenomenon, additional information on the structure of the flow-field inside the nozzle and vorticity enhancement may be obtained from Tipnis [19].

\section{References}

[1] P. M. Sforza, M. H. Steiger, N. Trentacoste, "Studies on threedimensional viscous jets", AIAA Journal, Vol. 4, pp. 800-806, 1966.

[2] N. Trentacoste, P. M. Sforza, "Further experimental results for three-dimensional free jets", AIAA Journal, Vol. 5, pp. 885-891, 1967.

[3] P. M. Sforza, "A quasi-axisymmetric approximation for turbulent, three-dimensional jets and wakes", AIAA Journal, Vol. 7, pp. 1380-1383, 1969.

[4] Y. Tsuchiya, C. Horikoshi, T. Sato, "On the spread of rectangular jets”, Experiments in Fluids, Vol. 4, pp. 197-204, 1986.

[5] C.-M. Ho, E. Gutmark, "Vortex induction and mass entrainment in a small-aspect-ratio elliptic jet", Journal of Fluid Mechanics, Vol. 179, pp. 383-405, 1987.

[6] G. N. Abramovich, "On the deformation of the rectangular turbulent jet cross-section", International Journal of Heat Mass Transfer, Vol. 25, pp. 1885-1894, 1982.

[7] F. Hussain, H. S. Husain, "Elliptic jets. part 1. characteristics of unexcited and excited jets", Journal of Fluid Mechanics, Vol. 208, pp. 257-320, 1989.

[8] H. Viets, P. M. Sforza, "Dynamics of bilaterally symmetric vortex rings", Physics of Fluids, Vol. 15, pp. 230-240, 1972.

[9] J. R. Hertzberg, C.-M. Ho, “Time-averaged, three-dimensional flow in a rectangular sudden expansion", AIAA Journal, Vol. 30, pp. 2420-2425, 1992.

[10] K. B. M. Q. Zaman, "Axis switching and spreading of an asymmetric jet: the role of coherent structure dynamics", Journal of Fluid Mechanics, Vol. 316, pp. 1-27, 1996.

[11] F. F. Grinstein, "Entrainment, axis switching, and aspect-ratio effects in rectangular free jets", $4^{\text {th }}$ Shear Flow Control Conference, Snowmass Village, CO, USA, AIAA-1997-1875, 1997.

[12] P. Bradshaw, "Turbulent secondary flows", Annual Review of Fluid Mechanics, Vol. 19, pp. 53-74, 1987.

[13] A. A. Sfeir, "The velocity and temperature fields of rectangular jets", International Journal of Heat and Mass Transfer, Vol. 19, pp. 1289-1297, 1976.

[14] A. A. Sfeir, "Investigation of three-dimensional turbulent rectangular jets”, AIAA Journal, Vol. 17, pp. 1055-1060, 1979.

[15] A. Krothapalli, D. Baganoff, K. Karamcheti, "On the mixing of a rectangular jet”, Journal of Fluid Mechanics, Vol. 107, pp. 201220, 1981.

[16] F. F. Grinstein, "Vortex dynamics and entrainment in rectangular free jets", Journal of Fluid Mechanics, Vol. 437, pp. 69-101, 2001.

[17] F. R. Menter, "Two-equation eddy-viscosity turbulence models for engineering applications”, AIAA Journal, Vol. 32, pp. 1598$1605,1994$.

[18] W. Rodi, Turbulence Models and their Applications in Hydraulics - A State of the Art Review, International Association for Hydraulic Research, Rotterdamseweg 185 - P.O.Box 177, 2600 MH Delft, The Netherlands, $2^{\text {nd }}$ edition, 1984.

[19] T. J. Tipnis, "Effects of upstream nozzle geometry on rectangular free jets”, PhD Thesis, Cranfield University, 2010.

[20] J. J. Miau, T. S. Leu, J. H. Chou, S. A. Lin, C. K. Lin, "Flow distortion in a circular-to-rectangular transition duct", AIAA Journal, Vol. 28, pp. 1447-1456, 1990.

[21] D. O. Davis, F. B. Gessner, "Experimental investigation of turbulent flow through a circular-to-rectangular transition duct", AIAA Journal, Vol. 30, pp. 367-375, 1992. 